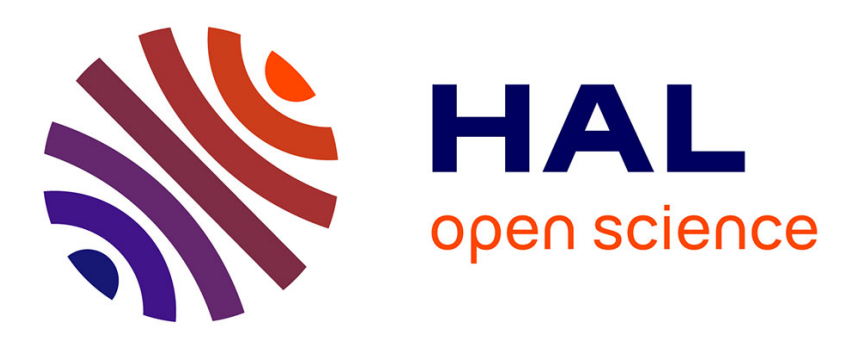

\title{
The role of the endocannabinoid system in autism spectrum disorders: Evidence from mouse studies
}

Susanna Pietropaolo, Luigi Bellocchio, Inés Bouzón-Arnáiz, Benjamin K Yee

\section{To cite this version:}

Susanna Pietropaolo, Luigi Bellocchio, Inés Bouzón-Arnáiz, Benjamin K Yee. The role of the endocannabinoid system in autism spectrum disorders: Evidence from mouse studies. Mirolyuba Ilieva; Way Kwok-Wai Lau. Autism, 173, pp.183-208, 2020, Progress in Molecular and Translational science, 10.1016/bs.pmbts.2020.04.016 . hal-03077547v2

\section{HAL Id: hal-03077547 \\ https://hal.science/hal-03077547v2}

Submitted on 26 Mar 2021

HAL is a multi-disciplinary open access archive for the deposit and dissemination of scientific research documents, whether they are published or not. The documents may come from teaching and research institutions in France or abroad, or from public or private research centers.
L'archive ouverte pluridisciplinaire HAL, est destinée au dépôt et à la diffusion de documents scientifiques de niveau recherche, publiés ou non, émanant des établissements d'enseignement et de recherche français ou étrangers, des laboratoires publics ou privés. 


\title{
THE ROLE OF THE ENDOCANNABINOID SYSTEM IN AUTISM SPECTRUM DISORDERS: EVIDENCE FROM MOUSE STUDIES
}

\author{
Susanna Pietropaolo ${ }^{1,2}$, Luigi Bellocchio ${ }^{2,3}$, Ines Bouzon-Arnaiz ${ }^{4}$, Benjamin K. \\ Yee $^{5}$ \\ ${ }^{1}$ University of Bordeaux, 146 rue Léo Saignat, 33077 Bordeaux Cedex, France \\ ${ }^{2}$ CNRS, INCIA, UMR 5287, Bat B2, Allée Geoffroy St. Hilaire, CS 50023, 33615 Pessac cedex, France \\ ${ }^{3}$ INSERM, U1215 NeuroCentre Magendie, 146 rue Léo Saignat, 33077 Bordeaux Cedex, France \\ ${ }^{4}$ University of Pompeu-Fabra, Barcelona, Spain \\ ${ }^{5}$ Department of Rehabilitation Sciences, Faculty of Health \& Social Sciences, The Hong Kong \\ Polytechnic University, Hong Kong
}

\section{Correspondence to:}

Susanna Pietropaolo,

Bordeaux University and CNRS, INCIA, UMR 5287,

Bat B2, Allée Geoffroy St. Hilaire,

CS 50023, 33615 Pessac cedex,

France

Tel : +33 (5) 4000-8748

Fax : +33 (5) 4000-8743

Email : susanna.pietropaolo@u-bordeaux.fr 


\section{ABSTRACT}

Recent years have seen an impressive amount of research devoted to understanding the etiopathology of Autism Spectrum Disorder (ASD) and developing therapies to treat this complex syndrome. This work has been largely based on the employment of rodent models, based on a multitude of genetic and environmental manipulations. Unfortunately, the task of identifying suitable candidates playing a relevant etiopathological role in ASD is highly challenging, due to a number of problems specifically affecting the research in this field. These include (i) the heterogeneity of ASD itself, including several disorders, (ii) the complexity of ASD etiology, involving a plethora of genetic and environmental factors, (iii) the presence of a huge variety of rodent models, without a universal consensus on their validity. Here we highlight how the endocannabinoid system (ECS) emerges within this complex context as a valid therapeutic target and etiopathological factor in ASD. The multiple roles of the ECS in modulating neuronal functions are described, to underline the overall relevance of this system. We summarize then the most recent results showing alterations of the ECS in rodent models of ASD (with an emphasis on mouse studies), and demonstrating ASD-like behaviours in mice with altered ECS, induced either by genetic or pharmacological manipulations. We also give a critical overview of the most relevant advances in designing treatments and novel mouse models for ASD targeting the ECS, highlighting the approaches that seem more promising to simplify the complex field of research on treatments for ASD.

Keywords: Autism, social interactions, mouse models, cannabinoids, CB1, developmental disorders 


\section{ANIMAL MODELS OF AUTISM SPECTRUM DISORDER (ASD)}

Autism Spectrum disorder (ASD) is probably among the pathologies of the central nervous system with the highest number of attempts of diagnostic definition. The criteria for diagnosing autism, as the disorder was originally called, were firstly proposed by Kanner [1], subsequently refined by Rutter [2] and then reformulated several times in the Diagnostic and Statistical Manual of Mental Disorders (DSM). According to the original definitions, a child can be ascertained for autism when the onset of the disturbance, characterised by pervasive social impairment and deficits in language and/or communication, occurred before 30 months, in the absence of the delusions and hallucinations typically found in schizophrenic patients [3]. The subsequent revisions of the DSM tended to isolate pervasive developmental disorders, highlighting that autistic symptomatology is instead not confined to early childhood, but persists afterwards [4]. Overall, the basic diagnostic criteria of autism include the following triad: social dysfunction, qualitative deficits in verbal/nonverbal communication and a restricted range of interests associated with the occurrence of repetitive behaviours. These criteria were substantially maintained in the more recent editions of DSM, although the first and second domains tended to be merged into a more general field of social/communication deficits.

Introducing the concept of Spectrum" has drastically changed our view of autistic pathology. ASD is now defined as a single disorder that includes pathologies that were previously considered separate, i.e., autism, Asperger's syndrome, childhood disintegrative disorder and pervasive developmental disorder not otherwise specified [5]. The term "spectrum" also refers to the wide range of symptoms and degrees of severity characterizing ASD patients, underlining the huge variability affecting the expression of both core (i.e., the autistic "triad") and additional symptoms [6]. Language deficits and delays are for example completely absent in patients with Asperger's syndrome, as is mental retardation. Also, ASD patients may either underreact or overreact to sensory 
stimuli, and they may show highly variable intelligence levels, from deficits to unevenly developed cognitive skills.

This impressively high heterogeneity of ASD is therefore related to the fact that it has been somehow artificially defined as a single disorder, but it is in reality a combination of divergent disorders, all having in turn variable symptoms. The complex nature of ASD and of its definition obviously complicates the designing of animal models of this disorder. The latter task is already made difficult by the unknown etiology of the disorder, including complex multigenic and environmental factors, with specific contributions and underlying mechanisms that still remain undefined. Furthermore, the lack of biomarkers of autistic pathology directs all the possible research focus towards the behavioural validation of its animal models. Assessing the face validity of animal models of ASD is also a challenging task, as some of the core behavioural symptoms are not easily modelled in rodents, especially in mice. Hence, a large body of recent research has focused on developing more appropriate methods to assess the core ASD-like behaviours in mice. Social interest for example has been assessed in juvenile and adult animals using social preference tasks, such as the automated three compartment test [7], or direct interaction tests, evaluating juvenile play, affiliative behaviours, or aggression. Furthermore, communication can also be assessed in laboratory mice, since they emit USVs in social contexts at multiple stages of their life $[8,9]$, including the first postnatal days $[10,11]$, but also during adult social interactions, namely male-female and female-female encounters $[12,13]$. The last core symptom of the autistic triad, i.e., the occurrence of repetitive behaviours and insistence on the sameness, has also been evaluated in laboratory mice, either through indirect measures of behavioural flexibility [14], e.g., assessing reversal learning in different types of mazes, or through the assessment of home-cage or novelty-induced behavioural stereotypies, e.g. excessive self-grooming. Additional ASD-relevant symptoms such as anxiety, sensory abnormalities, hyper-activity, and cognitive alterations can be instead tested in mice with well-established and standardized behavioural protocols. 
To face the challenges of basic research on ASD, a promising approach seems to consist of discarding any unitary explanation of ASD, as suggested by some authors [15], and trying to model the symptoms, rather than the whole disorder. The definition of the precise criteria that a valid model for ASD should fulfil is still the subject of an intense debate [16]: the most obvious point of view considers an ideal animal model as the one mimicking all core autistic features in association with some of the secondary ones. Nonetheless, the most realistic research approaches state that a useful animal model should present behavioural features that resemble at least one of the autistic core symptoms, thus emphasizing the importance of "incomplete" mouse models of ASD, as already suggested for other disorders [17]. The most generally used criterion considers a mouse model as valid if it presents alterations in the three core domains affected in ASD, i.e., social interaction, communication, and behavioural flexibility/repetitive behaviours $[9,16,18]$. The presence of additional behavioural abnormalities, such as hyper-activity, memory deficits or anxiety, may increase the validity of the model, but is not required [19].

As reviewed in several articles [e.g., [19-22]], a huge variety of mouse models have been proposed in the last decades, based on very different approaches. One strategy is based on identifying mouse strains that spontaneously present some major ASD-like symptoms, such as low sociability, poor communication abilities, and high levels of inflexible behaviours. The best-known example of this approach is the BTBR strain [23, 24], widely employed in ASD research and proposed as a tool to identify novel genes involved in the control of social behaviours. The main disadvantages of this model are (i) the problem of identifying an appropriate control strain and (ii) the possibility that strain-specific phenotypes of emotionality may explain the supposed ASD-like behavioural profiles [25].

Other mouse models rely on the exposure (mostly prenatally) to adverse environmental factors $[26,27]$, such as teratogens and viral infections, suspected to contribute to the aetiology of ASD on the basis of epidemiological data. A well-known example of this approach comes from the 
valproic acid (VPA) model, developed mainly in rats $[28,29]$, and secondarily in mice $[30,31]$. This approach has the advantage of having a sort of "construct validity", although incomplete, but it has the main drawback of overlooking the major role of genetic factors in ASP etiopathology. Indeed, another approach consists in manipulating the mouse genome to import into the animal model some of the genetic alterations associated with ASD, e.g., gene deletion/ mutations, or chromosomal abnormalities. The validity of this experimental strategy also has some problems, i.e., the heterogeneity of the genetic alterations known to be somehow involved in ASD, including basically almost all genes affecting neuronal function and development [32]. These genes code for molecules involved in: i) chromatin remodelling and regulation of transcription (e.g., MeCP2, FMR1), ii) actin cytoskeleton dynamics (TSC1, TSC2, NF1) iii) synaptic scaffolding proteins (e.g., SHANK3), iv) receptors and transporters (e.g., GRIN2A, GRIK2, GABAR, SLC6A4, SLC25A13, OXTR, AVPR1), v) second-messenger systems (PRKCB1, CACNA1C, NBEA), vi) cell adhesion (e.g.,neuroligin NLGN3, NLGN4), and vii) secreted proteins (e.g., RELN, LAMB1) (reviewed in [33]). Such list serves only to provide an indicative overview, as the number of implicated genes is supposed to be many-fold greater [34].

The role of all these genes in brain development is at the basis of a recent hypothesis on autistic etiology, i.e., the so-called "many genes, common pathways" [35], emphasizing the role of defective synaptic functioning and abnormal brain connectivity in ASD, both leading to altered information processing. One somewhat paradoxical finding about ASD is that, although there is proof that its etiopathogenesis is the result of concomitant mutations in multiple loci, there is a growing list of genes whose disruption alone is sufficient for the whole autistic phenotype to occur. Based on such evidence, mouse models of single gene mutations may therefore be informative concerning the underlying mechanism(s) leading to ASD [36].

The last approach used to design animal models of ASD is indeed based on focusing on other developmental pathologies featuring ASD-like traits, but with a defined, single genetic cause (and 
well-established mouse models). A widely used example of this approach is provided by the Fmr1-KO mouse line [37], modelling the most common monogenic cause of ASD, that is Fragile $X$ syndrome (FXS), a X-linked disorder due to the lack of FMRP protein, coded by the FMR1 gene [38]. Fmr1-KO mice showed ASD-like deficits, i.e., reduced social interaction and social memory, deficits in reversal learning and US communication alterations, as reviewed in [39]. A criticism to this research strategy comes from the consideration that the genetic mutations induced in these models are not invariably present in ASD patients and may therefore be valid in modelling only a specific subset of patients [40]. Nonetheless, recent research on pre-clinical models increasingly supports the view that investigating the consequences of single gene disruption may constitute a valuable tool for understanding ASD $[8,9]$, a research approach that we have also followed in a major part of our recent research work [41].

In this complex and somehow undefined field of research, basically all these approaches to model ASD in laboratory mice have been employed to try to identify potential therapeutic targets to treat ASD. In the present chapter we will indeed take advantage of examples of these different strategies to design mouse models of ASD to summarize the experimental evidence supporting the role of the endocannabinoid system (ECS) in the etiopathology of ASD. To this end, we will first review studies showing abnormalities in the functionality of ECS in mouse models of ASD, including examples from the approaches described above. These will include rodent models based on strain differences (the BTBR mouse), prenatal exposure to environmental agents (the VPA model), candidate genes (NLGN-3 mutant mice), or monogenic developmental pathologies (the Fmr1-KO mouse). At the same time, we will summarize data showing the correction of ASD-like behaviours in these types of mouse models following pharmacological modulation of the ECS, thus suggesting potential novel pharmacological approaches to ASD. Finally, we will evaluate the possibility of inducing ASD-like behaviours in mice through pharmacological or genetic manipulations of the ECS, thus discussing potential novel models for ASD. 


\section{UNDERSTANDING THE ETIOPATHOLOGY OF ASD: THE ROLE OF THE ENDOCANNABINOID SYSTEM}

Understanding the etiology of ASD is an extremely challenging task, as this disorder is due to a combination of genetic and environmental factors, acting through a variety of complex mechanisms, e.g., imbalances of excitation and inhibition as well as hypo/hyper-connectivity [42]. In addition, as already mentioned, the genetic causes of ASDs are highly heterogeneous, with hundreds of ASDassociated genes [32]. The definition of the possible pathogenic mechanisms is difficult to be achieved, as they include multiple genetic mutations, their combinations, and their interactions with genetic factors [43]. The investigation of the combination of genetic risk factors with environmental adversity has received increasing attention in ASD research [15], as it seems a key to understand the marked inter-individual phenotypic differences observed in ASD patients [43, 44]. Recent studies have suggested that all this multitude of causal factors may functionally converge into a relatively smaller subset of functional pathways that may represent an easier and more promising target for basic research on ASD [45]. The functional convergence on particular signalling pathways and the shared synaptopathology of ASD raise the hope that similar therapeutic strategies may be effective for different forms of ASD which are related, but genetically distinct [45]. This "reductionist" approach is in line with the hypothesis of "many genes, common pathways" [35] mentioned before, underlying the convergence of multiple causal factors onto common mechanisms, leading to the altered synaptic functioning and defective information processing observed in ASD.

Understanding the etiopathology of ASD is of obvious relevance to identify therapeutic targets to treat this complex disorder. Pharmacological therapies currently available for ASD patients are indeed limited to the treatment of some relatively minor symptoms, including anxiety, irritability, aggressiveness and epilepsy. The most common drug classes used include antipsychotics, anxiolytics and anticonvulsants, i.e., therapies that do not act on any causal factor of the disorder and have heavy side effects, especially in children. Therefore, the most recent approach of animal research on pharmacological therapies for ASD is to focus on the common mechanisms potentially underlying 
ASD etiopathology to identify molecular targets for powerful pharmacological interventions. Several studies following this approach have underlined for instance the leading role of dysregulation of activity-dependent protein synthesis in the pathogenesis of this disorder. Protein synthesis is in fact modulated by most "ASD genes" either intrinsically or indirectly by impairing synaptic function. Compounds acting on this process have been proposed as potential treatments for ASD, e.g., rapamycin inhibiting the mTOR pathway, or mGluR1/5 antagonists to inhibit metabotropic glutamate receptor-related modulation of synaptic activity (an extensive description of the effects of these and similar compounds can be found in previous reviews [e.g., 46, 47]). Nonetheless, pharmacological treatments with sufficient pre-clinical and clinical efficacy are still lacking.

In this context supporting the search for common causal pathways for ASD, the endocannabinoid system (ECS) seems a promising candidate to play a role in the etiopathology of this disorder and to provide a novel target of therapeutic interest. The ECS has recently emerged as an important modulator of neuronal functions, as demonstrated by the abundance of the cannabinoid CB1 receptors in the brain [48]. These are particularly enriched in cortex, hippocampus, amygdala, and cerebellum, i.e., brain areas which are strongly involved in the development of ASD. Furthermore, endocannabinoid signaling also regulates synaptogenesis and neuronal interconnectivity during development [49], processes whose defects are considered to be key mechanisms underlying ASD pathology [42].

CB1 receptors are located pre-synaptically in both GABAergic and glutamatergic neurons [50] where they are activated by their endogenous ligands, i.e., the endocannabinoids (ECs): anandamide (AEA) and 2-arachidonoyl-glycerol (2-AG). These endogenous cannabinoids are produced at postsynaptic site by membrane depolarization or activation of metabotropic receptors [50].

Indeed calcium elevation generated by these two type of events is able to trigger plasma membrane lipid remodeling which generates as final products AEA, by the biosynthetic enzyme $\mathrm{N}$ - 
acyl phosphatidylethanolamine-specific phospholipase D (NAPE-PLD) and 2-AG, by the Diacylglycerol lipase alpha (DAGL-a) [50]. AEA and 2-AG travel backwards reaching CB1 receptors located at presynaptic site, where by triggering several intracellular pathway are able to reduce intracellular calcium, resulting in an inhibition of neurotransmitter release [50]. Then, endocannabinoids are reuptake and degraded by fatty acid amide hydrolase (FAAH), the main degrading enzyme for AEA, and by monoacyl-glycerol lipase (MAGL), the main enzyme for 2-AG. The ECS differs from classical neurotransmitter systems for the fact that there is no constitutive tone, but on-demand production of ECs, CB1 receptor activation followed by immediate ECs degradation [50]. Several pharmacological tools are available for studying the ECS: indeed the system can be activated by direct administration of CB1 agonist [e.g., cannabinoid Delta(9)-tetrahydrocannabinol (THC)], as well as more specific inhibitors of AEA and 2-AG reuptake and degradation (which will results in a more selective activation, by potentiating the activity of the system only when and where is active) [51]. On the other hand, the ECS can be blocked by selective antagonists of CB1 receptor [e.g., rimonabant (SR141716A)], as well as ECs' biosynthetic enzymes inhibitors [50]. It is worth to mention also the development in the last years of constitutive and cell type specific knockout mouse models for CB1 receptor [52, 53], NAPE-PLD, DAGLa, FAAH and MAGL (reviewed in [54]), which have provided more in deep mechanism for ECS-mediated regulation of a plethora of brain and peripheral functions [55].

ECS is therefore a neuromodulatory network of lipid signaling pathways, including the ECs, together with their receptors and the associated metabolic enzymes [56]. Although ECs exert their central effects mainly through the cannabinoid type 1 receptor (CB1r), they also interact with the other G-protein coupled cannabinoid type 2 receptor (CB2r), mostly involved in inflammatory processes and in the neuromodulation of immune system [57, 58]. Additionally, they can also interact with non-CB1/CB2 targets, e.g., the transient receptor potential vanilloid type 1 (TRPV1) channel, peroxisome proliferator-activated receptor (PPAR)-alpha, and PPAR-gamma as well as the G protein-coupled receptor GPR55 [59]. The etiopathological role of alterations in ECS functionality has been already suggested for several psychiatric [60] and neurological disorders [61], e.g., schizophrenia, anxiety, depression, neurodegenerative diseases, epilepsy [55]. This is not surprising, considering the major impact of ECS in the modulation of neuronal and synaptic function as well as of brain development. 
Indeed, a clear contribution of ECS has been demonstrated on behavioural functions that are altered in several neuropathologies, including ASD. These include cognitive and emotional abilities, circadian rhythms, epileptic seizures and social interaction (e.g., [53, 62-64]). Beside this indirect evidence, recent findings have provided a more direct link between ECS functionality and ASD. Human neuroimaging studies have for instance demonstrated that variations of the cannabinoid receptor gene are involved in the modulation of social reward responsivity, specifically in the striatal response to happy faces [65], a function which is specifically altered in ASD patients. Post-mortem analyses showed reduced expression of CB1 receptors in several areas of ASD brains [66], while recent studies reported that AEA plasmatic concentrations were significantly lower in ASD children compared to controls [67], in the absence of changes in blood levels of 2-AG [68]. It is still not clear whether the reduced AEA levels could be due to a reduced activity of NAPE-PLD, the enzyme that synthesizes AEA or increased activity of FAAH, the enzyme that degrades them. Furthermore, rare genetic variants in the core endocannabinoid system genes (e.g., CNR1, CNR2, DAGLA, MGLL and FAAH) were identified in molecular testing data from a large sample of patients with a broad spectrum of neurological disorders, showing that heterozygous rare variants in DAGLA were significantly associated with seizures and ASD [69]. In addition, increased expression of CB2 receptors has been described in blood monocytes of ASD patients [70], suggesting a role of ECS in mediating the immune alterations of ASD pathology. CB2r is indeed able to modulate development, migration, proliferation, and effector functions of immune cells [71], and alterations in immune system have been reported in ASD patients $([72,73]$. Based on this large body of evidence from ASD patients, several studies have employed animal models in the last years to better evaluate the role of ECS in the etiopathology of ASD.

\section{ALTERATIONS OF ECS IN ANIMAL MODELS OF ASD: TOWARDS NOVEL THERAPEUTIC}

\section{APPROACHES}


Alterations in ECS functionality have been described in several animal models of ASD (reviewed also in [74]), based on the approaches described in the first section of the present chapter. Here we will therefore review the studies investigating ECS in ASD rodent models, including available examples from approaches based on monogenic diseases (the Fmr1-KO mouse), strain differences (the BTBR model), candidate genes (the example of NLGN mice), and environmental toxins exposure (the VPA model).

The ASD model that has been the most extensively investigated in relation to ECS is the Fmr1-KO mouse, since this is also a model of another major (but monogenic) developmental disorder associated with ASD, that is FXS [39]. As FXS is also a developmental pathology lacking therapeutic targets, a large amount of research has been devoted on the role of ECS in FXS on its own. Alterations in the ECS functionality have been demonstrated in Fmr1-KO mice by several studies, highlighting an imbalance in EC signalling and suggesting the therapeutic relevance of its correction by targeting different components of the ECS, including 2-AG, $A E A$, cannabinoid $C B 1$ and $C B 2$ receptors. First, it was demonstrated that the coupling between hippocampal mGluR activation and eCB mobilization was enhanced in Fmr1-KO mice, in the absence of alterations in the expression of cannabinoid CB1R [75]. Second, enhanced striatal activity of diacylglycerol lipase (DAGL) was reported in these KOs [76], and enhancing 2-AG signalling through administration of JZL184, a pharmacological inhibitor of the 2-AG degrading enzyme MAGL, corrected some of the mutants' behavioural alterations, e.g.,hyperactivity and anxiety abnormalities [77]. We also obtained results supporting altered ECS functionality in Fmr1-KO mice, as demonstrated by their different behavioural response to acute pharmacological challenge of the ECS, using either the CB1r antagonist rimonabant, or an agonist, WIN 55,212-2. On one hand, Fmr1-KOs showed a similar reduction in fasting-induced food intake, i.e., a classical effect of acute rimonabant administration $(3 \mathrm{mg} / \mathrm{Kg}$, i.p.) which has been shown to depend on peripheral CB1 [78]. On the other hand, mutant mice showed a marked reduced response to the acute injection of WIN 55,212-2 [79], as assessed by the tetrad 
experiment (i.e., assessing the hypolocomotion, hypothermia, analgesia and catalepsy typically induced by a $3 \mathrm{mg} / \mathrm{Kg}$ dose of this CB1 agonist).

When the impact of pharmacological manipulations of $C B$ receptors was studied more in details in Fmr1-KO mice, the effects seem to critically depend on the type of behavioural alterations analyzed. While CB1R blockade (also through genetic approaches, i.e., generation of double knockouts for CB1 and Fmr1) eliminated the cognitive impairments, nociceptive alterations and susceptibility to audiogenic seizures, pharmacological blockade of CB2R rescued anxiety abnormalities [80]. As some of these beneficial effects were accompanied by normalization of overactivated mTOR signaling and were observed also following pharmacological inhibition of mTOR or mGluR5 in the same Fmr1-KO mice, some authors have suggested that mTOR pathway may represent a key mechanism to explain the role of ECS in ASD [80]. The same authors have supported the potential therapeutic applications of rimonabant for FXS and ASD, although beneficial effects were described only on the memory deficits of Fmr1-KO mice [81]. Despite the efforts of considerably lowering the doses of this CB1 antagonist in Fmr1-KO animals [81], it is unlikely that strategies directly targeting CB1 may still be actively investigated, due to the high risk of severe adverse effects. A stronger interest has been instead devoted to target AEA levels, always using the Fmr1-KO model. Administration of inhibitors of FAAH, thus increasing AEA levels, has shown to eliminates the cognitive [82] and social deficits of Fmr1-KO mice [83].

A key role of AEA levels in ASD pathology was suggested also by the BTBR mouse model, a strain characterized by low levels of sociability, and therefore proposed as a model for ASD [23, 24]. In this mouse line URB597, a FAAH inhibitor, rescued the social impairments, and this effect was independent from changes in anxiety-levels and was prevented by CB1r blockade [83]. BTBR mice also showed an enhancement of CB1receptor functionality in cortical [84] and hippocampal areas [85]. Some authors suggested that CB1r up-regulation could be related to the hyper-responsiveness of the HPA axis characterizing these mice [85]. The role of oxytocin was instead underlined by others 
[83], as this neuropeptide drives anandamide mobilization in some brain areas in the mouse, e.g., the nucleus accumbens (NAc), a brain structure that regulates motivated behaviour [86]. Interestingly, AEA mobilization in this brain area is potentiated by social contact and inhibited by social isolation in mice [86]. To our knowledge, only one study investigated the behavioural response of BTBR to CB1 agonists, leading to results similar to those we described in Fmr1-KO mice, i.e., showing a reduced sensitivity to the sedative effects of high doses of THC [87].

While evidence from Fmr1-KO and BTBR mice overall tend to converge on similar alterations in ECS functionality, findings from the "candidate gene" model based on the cell-adhesion molecule neuroligin NLG3 mutations seem to diverge. These mutants showed reduced CB1-dependent modulation of GABAergic transmission in the hippocampus [88] and cortex [89]. A partial rescue of LTD was obtained by exogenous activation of cannabinoid CB1 receptors, thus suggesting a reduced cannabinoid drive in these mutants [90]. In line with these findings, beneficial behavioral effects, i.e., a significant reduction in aggressive behaviour, was described following the administration of nonsedating doses (0.3 and $1.0 \mathrm{mg} / \mathrm{kg}$ ) of CB1r agonists in NL3 mice [91].

Finally, evidence for the involvement of ECS in ASD was obtained from an environmental based model, i.e. the VPA model $[28,29]$, although to our knowledge all studies on this issue were conducted in rats. Rats prenatally exposed to VPA displayed altered expression of phosphorylated CB1 receptor in the amygdala, hippocampus and dorsal striatum [92] [93]. Although no changes in baseline concentrations of AEA and 2-AG were observed [100], enhancing AEA signalling through inhibition of its degradation was able to mitigate the behavioral phenotype induced by prenatal VPA exposure [92] [94], as previously described here for some mouse models of ASD.

In conclusion, the data from mouse models support the presence of alterations in the functionality of the ECS, involving multiple components of the systems, sometimes with different modalities according to the considered model. Nonetheless, the role of ECs and of AEA in particular, emerges as a common element across multiple models (including ASD patients) and therefore a 
potential central factor in the etiopathology of ASD. Clinical Studies showing lower plasmatic levels of AEA in autistic patients $[67,68]$ suggested that circulating endocannabinoid levels could be used as stratification biomarkers that identify clinically significant subgroups within the autism spectrum. Identifying biomarkers is in general crucial to provide diagnostic and prognostic information, and monitor responses to treatments. This is specifically imperative in the case of ASD because of the high complexity and heterogeneity of the disorder and the importance of early diagnosis and early behavioral intervention. Circulating ECs might be used to identify a biologically homogeneous subgroup of ASD, and optimize the development of novel drugs that target specific core symptoms of ASD. They could also contribute to predict autism risk prior to the onset of behavioral abnormalities and to initiate early interventions, since AEA levels appeared inversely correlated with age in ASD patients $[67,68]$.

Taking into account the relevance of ECs in ASD, it is not surprising that some phytocannabinoids, such as Cannabidiol (CBD), have been suggested as therapeutic strategies for this disorder [95]. As CBD has low affinity for CB1 receptors, it has been indeed hypothesized that its therapeutic effects could be due to the inhibition of FAAH [56] and therefore a compensation of the low levels of AEA found in ASD patients [68]. Preliminary data showed that oral CBD administration improved symptoms such as hyperactivity, self-injurious behaviours, anxiety and sleep problems in ASD children [96]. Furthermore, clinical studies have highlighted the therapeutic potential of CBD for ASD and cognitive symptoms in Fragile $X$ patients, in the absence of relevant adverse effects $[97,98]$.

The high interest in CBD and its derived compounds, e.g., cannabidivarin (CBDV), stems from the fact that these molecules do not directly act on the major CB1r, thus avoiding the noxious psychotropic effects typical of both THC or rimonabant). Furthermore, CBDV has powerful antiinflammatory properties [99], probably due to its action as agonist of transient potential (TRP) channels [100]. Hence, several preclinical studies have been conducted to evaluate the therapeutic efficacy of CBDV in animal models of ASD. For instance, chronic systemic administration of CBDV 
rescued the social deficits as well as the brain alterations of MeCP2-308 mice [101], a validated model of Rett syndrome [37], a monogenic disorder due to the lack of methyl-CpG-binding protein-2 (Mecp2) and characterized by ASD deficits. Mice carrying null mutation for Mecp2 [38], as well as various polymorphisms found in Rett patients [39], display ASD-like phenotypes, e.g., altered USV emission at infancy [40], social and cognitive deficits at adulthood [41]. CBDV also eliminated the cognitive deficits of Mecp2 mice, concomitantly normalizing the upregulation of CB1 $r$ and CB2 $r$ brain, thus further supporting the role of ECS in this ASD-related developmental disorder [102]. CBDV also rescued the cognitive, social and repetitive alterations shown by VPA rats, restoring hippocampal endocannabinoid signaling and neuroinflammation induced by prenatal VPA exposure [103]. Our unpublished results from Fmr1-KO mice also supported the efficacy of CBDV chronic treatments for ASD-like behavioural alterations, although the early timing of intervention appeared to be crucial. The efficacy of CBDV was in fact markedly higher when the treatment was administered starting at weaning than at adulthood in our Fmr1-KO mice, suggesting that sensitive therapeutic windows for ECS manipulations should be taken into account.

\section{ASD-LIKE PHENOTYPES INDUCED BY MANIPULATIONS OF THE ECS: RELEVANCE FOR DESIGNING}

\section{ANIMAL MODELS OF ASD}

The recent predominant research focus on genetic mouse models of ASD has somehow attenuated the interest in pharmacological approaches, though they may provide complementary precious information on the etiopathology of this disorder. Evaluating the ASD-like behavioural effects of pharmacological manipulations of the ECS in wild-type rodents can in fact contribute to shed light on the involvement of this complex neuromodulatory system in this developmental disease. The interest in the effects of cannabinoids on social behaviours has ancient roots, since psychotropic cannabinoids such as marijuana and hashish have been utilized for centuries as social recreational drugs, as well as euphoria-inducing compounds. As mentioned before, the discovery of CB1 receptor started to shed light on the molecular mechanism underlying the neurophysiological and behavioral 
effects of these cannabinoids, acting as CB1r agonists. Cannabis intoxication has been shown in humans and laboratory animals models to share several symptoms with ASD, such as cognitive and somatosensory impairment, social withdrawal and repetitive behavior [104]. However little is still known on the effects of recreational, non-intoxicating doses of cannabinoids on ASD-relevant behaviors, i.e., social behavior. In humans, THC has been shown to modulate the perception of social threats in a recent functional magnetic resonance imaging study [105], since recreational doses of THC significantly reduced amygdala reactivity to social signals of threat (without affecting activity in primary visual and motor cortical areas).

To date, the evaluation of the behavioral effects of acute (non-intoxicating) administration of crude marijuana estracts, pure THC or synthetic cannabinoids suspensions (e.g., WIN 55,212-2, [106]) yelded to contradictory results in mice, displaying either pro or antisocial effects (or sometimes aggression), according to the dose and the type of tests employed [107-111]. One reason for this discrepancy might be due to the differential expression and activation of CB1 receptor in different neuronal populations. Indeed an elegant series of studies that shed light on the biphasic effects of THC on both feeding and anxiety $[112,113]$ showing that opposite effects of different doses were mediated by activation of CB1 receptors on different neuronal populations, either glutamatergic or GABAergic $[112,113]$. A similar scenarion may apply also to the effects of THC on sociability, as suggested by recent studies on conditional knockout mice for CB1rin different neuronal types, showing abnormalities in different aspects of social behaviors [114-116]. A pharmacological approach to activate ECS that seems more straightforward than admininstration of CB1r agonists consists of strengthening endogenous endocannabinoids' signalling, for instance by enhancing AEA brain levels (as already mentioned in this chapter). The administration of AEA hydrolysis inhibitors (e.g.,URB597) either systemically or directly into brain limbic areas (e.g., amygdala and nucleus accumbens), has been reported for instance to increase social activity in rats [63] and reduced the anxiety induced by social defeat in mice [117]. 
While activating the ECS seems to induce controversial effects on social behaviours, the blockade of CB1 signaling is universally recognized as an « anti-social » treatment in animal research, and could therefore be instrumental in inducing ASD-like phenotypes. Systemic administration of the CB1r blocker AM251 in adult male capuchin monkeys induced a decrease in social and an increase in self-directed behaviors, as well as resistance to aversive memory extinction, without concomitant changes in the subjects' locomotor activity or vigilance behavior [118]. AM251 was shown to decrease social interest also in rats [119], and to reduce the preference for social context in mice [86], where it also altered social memory [120]. In line with these studies, we have investigated the ability of the CB1 antagonist rimonabant of inducing ASD-like behaviors in wild type B6 mice, i.e., the background that is mostly used to engineered the Fmr1-KO model. We evaluted the effects of an acute injection of increasing doses of rimonabant on the direct social interaction with an adult female, a test where Fmr1-KO mice showed an altered phenotype. As illustrated in Fig.2, Rimonabant reduced the levels of affiliative behaviors, in particular of anogenital sniffings, and the number of ultrasonic vocalizations (USV), while it increased those of self-grooming, i.e., it induced an ASD-like phenotype similar to that of Fmr1-KOs. All these effects were observed in the absence of confounding changes in overall locomotor activity.

Interestingly, ASD-like behaviours were also described following the genetic blockade of CB1 receptors, thus suggesting the possible relevance of CB1-KO mice to study ASD. Impaired sociability [123] and social exploration [114, 115] was observed in these KOs (although they seemed dependent on the testing context [84] and the type of social stimulus [116]), as well as altered social memory [120]. While repetitive behaviours, such as enhanced levels of self-grooming, were not consistently observed in CB1-KOs [120, 124], we have recently described qualitative (but not quantitative) alterations in USV vocalizations in CB1-KO mice, both during development (i.e., in the first post-natal week) and at adulthood [125]. Overall, the severity of ASD-like phenotypes seems less marked when induced by the genetic rather than the pharmacological induction of CB1r blockade, an effect that may be due to processes of developmental compensation. Nonetheless, the importance of CB1-KO 
mice to study the role of ECS in ASD remains remarkable, especially because of the availability of KO in specific neuronal populations, helping to disentangle the impact of glutamatergic, gabaergic, serotonergic and dopaminergic pools of these receptors in the modulation of social behaviours [114$116,120,124]$.

\section{CONCLUSIONS AND PERSPECTIVES}

Clinical and pre-clinical data strongly support the involvement of the ECS in the etiopathology of ASD, and its relevance as a therapeutic target to design novel pharmacological treatments. Evidence from mouse models of ASD and pharmacological manipulations of the ECS in wild type animals highlight the importance of an imbalance in ECS signaling as a common potential etiopathological mechanism of this complex disorder.

ECS could therefore be considered as a good candidate to fit into the theory of ASD of "multiple genes, common pathways", also because of its major modulatory role of neuronal functions and brain development. In this latter respect, some authors have recently suggested that the impact of ECS in ASD could be specifically related to its modulation of brain developmental processes [126], an issue that has been poorly elucidated in the context of ASD models. The use of induced pluripotent stem cell derived neurons (iPSC) has already provided valuable insight into the pathogenesis of neurological disorders such as ASDs [127], and should therefore be applied to future studies to try to differentiate the relevance of ECS in abnormal brain development from mature synaptic functionality [126].

The complexity characterizing ASD etiopathology reflects somehow the complexity of the ECS itself. On one hand, the discovery of the relevance of endocannabinoids in ASD raised new possibilities for safe targeting the ECS to treat this disorder, as demonstrated by the evidence reviewed here supporting the therapeutic use of phytocannabinoids, such as CBD and CBDV. These compounds are promising because of their anti-inflammatory properties, and because they can enhance endocannabinoid levels without the adverse effects of CB1r direct modulation. On the other 
hand, our knowledge of the complexity of the ECS has further increased, complicating clinical approaches. The discovery that endocannabinoids can activate different novel receptors and that their metabolic pathways are often shared with other mediators has led to the inclusion of the ECS into an expanded signaling system, known as the endocannabinoidome [55]. This enlarged ECS, including several mediators that are biochemically related to the endocannabinoids, together with their receptors and metabolic enzymes, is likely to provide novel molecular targets of therapeutic relevance to be tested in future studies on ASD [55].

Finally, despite the large body of evidence supporting a role of ECS in ASD, we do not intend to support the view that this system, even with its high complexity, may provide a unitary explanation of ASD etiopathology. We are instead convinced, in agreement with previous authors, that multiple actors must play a concomitant role in mediating the complex pathogenesis of this developmental disorder $[15,128]$. Hence, beside the refinement of pharmacological manipulations targeting the ECS, future efforts should focus on designing therapeutic approaches combining multiple molecular targets [129], as already suggested by others [130].

\section{REFERENCES}

[1] L. Kanner, Autistic disturbances of affective contact, Nervous child 2 (1943) 217-250.

[2] M. Rutter, Concepts of autism: a review of research, J Child Psychol Psychiatry 9(1) (1968) 1-25.

[3] American psychiatric association, Diagnostic and statistical manual of mental disorders (DSM-III), Washington, DC, 1980.

[4] American psychiatric association, Diagnostic and statistical manual of mental disorders (DSM-IIIR), Washington, DC, 1987.

[5] American psychiatric association, Diagnostic and statistical manual of mental disorders (DSM-V), Washington, DC, 2000.

[6] I. Rapin, Autistic children: diagnosis and clinical features, Pediatrics 87(5 Pt 2) (1991) 751-60. 
[7] J.J. Nadler, S.S. Moy, G. Dold, D. Trang, N. Simmons, A. Perez, N.B. Young, R.P. Barbaro, J. Piven, T.R. Magnuson, J.N. Crawley, Automated apparatus for quantitation of social approach behaviors in mice, Genes, brain, and behavior 3(5) (2004) 303-14.

[8] M. Wohr, Ultrasonic vocalizations in Shank mouse models for autism spectrum disorders: detailed spectrographic analyses and developmental profiles, Neurosci Biobehav Rev 43 (2014) 199-212.

[9] M. Wohr, M.L. Scattoni, Behavioural methods used in rodent models of autism spectrum disorders: current standards and new developments, Behav Brain Res 251 (2013) 5-17.

[10] I. Branchi, D. Santucci, E. Alleva, Ultrasonic vocalisation emitted by infant rodents: a tool for assessment of neurobehavioural development, Behav Brain Res 125(1-2) (2001) 49-56.

[11] F.R. D'Amato, E. Scalera, C. Sarli, A. Moles, Pups call, mothers rush: does maternal responsiveness affect the amount of ultrasonic vocalizations in mouse pups?, Behav Genet 35(1) (2005) 103-12.

[12] T.E. Holy, Z. Guo, Ultrasonic songs of male mice, PLoS biology 3(12) (2005) e386.

[13] A. Moles, F. Costantini, L. Garbugino, C. Zanettini, F.R. D'Amato, Ultrasonic vocalizations emitted during dyadic interactions in female mice: a possible index of sociability?, Behav Brain Res 182(2) (2007) 223-30.

[14] S.S. Moy, J.J. Nadler, M.D. Poe, R.J. Nonneman, N.B. Young, B.H. Koller, J.N. Crawley, G.E. Duncan, J.W. Bodfish, Development of a mouse test for repetitive, restricted behaviors: relevance to autism, Behav Brain Res 188(1) (2008) 178-94.

[15] F. Happe, A. Ronald, R. Plomin, Time to give up on a single explanation for autism, Nat Neurosci $9(10)(2006)$ 1218-20.

[16] J.N. Crawley, Mouse behavioral assays relevant to the symptoms of autism, Brain Pathol 17(4) (2007) 448-59.

[17] R. Radde, C. Duma, M. Goedert, M. Jucker, The value of incomplete mouse models of Alzheimer's disease, European journal of nuclear medicine and molecular imaging 35 Suppl 1 (2008) S70-4. 
[18] J.N. Crawley, Designing mouse behavioral tasks relevant to autistic-like behaviors, Ment Retard Dev Disabil Res Rev 10(4) (2004) 248-58.

[19] S.S. Moy, J.J. Nadler, T.R. Magnuson, J.N. Crawley, Mouse models of autism spectrum disorders: the challenge for behavioral genetics, Am J Med Genet C Semin Med Genet 142C(1) (2006) 40-51.

[20] S. Banerjee, M. Riordan, M.A. Bhat, Genetic aspects of autism spectrum disorders: insights from animal models, Frontiers in cellular neuroscience 8 (2014) 58.

[21] A.L. Bey, Y.H. Jiang, Overview of mouse models of autism spectrum disorders, Current protocols in pharmacology / editorial board, S.J. Enna 66 (2014) 566 1-5 6626.

[22] J. Ellegood, J.N. Crawley, Behavioral and Neuroanatomical Phenotypes in Mouse Models of Autism, Neurotherapeutics : the journal of the American Society for Experimental NeuroTherapeutics $12(3)$ (2015) 521-33.

[23] H.G. McFarlane, G.K. Kusek, M. Yang, J.L. Phoenix, V.J. Bolivar, J.N. Crawley, Autism-like behavioral phenotypes in BTBR T+tf/J mice, Genes, brain, and behavior 7(2) (2008) 152-63.

[24] K.Z. Meyza, E.B. Defensor, A.L. Jensen, M.J. Corley, B.L. Pearson, R.L. Pobbe, V.J. Bolivar, D.C. Blanchard, R.J. Blanchard, The BTBR T+tf/J mouse model for autism spectrum disorders-in search of biomarkers, Behavioural brain research 251 (2013) 25-34.

[25] D. Oddi, W.E. Crusio, F.R. D'Amato, S. Pietropaolo, Monogenic mouse models of social dysfunction: Implications for autism, Behavioural brain research 251 (2013) 75-84.

[26] D. Dufour-Rainfray, P. Vourc'h, S. Tourlet, D. Guilloteau, S. Chalon, C.R. Andres, Fetal exposure to teratogens: evidence of genes involved in autism, Neuroscience and biobehavioral reviews $35(5)$ (2011) 1254-65.

[27] P.H. Patterson, Maternal infection and immune involvement in autism, Trends in molecular medicine 17(7) (2011) 389-94.

[28] J.L. Ingram, S.M. Peckham, B. Tisdale, P.M. Rodier, Prenatal exposure of rats to valproic acid reproduces the cerebellar anomalies associated with autism, Neurotoxicol Teratol 22(3) (2000) 31924. 
[29] T. Schneider, R. Przewlocki, Behavioral alterations in rats prenatally exposed to valproic acid: animal model of autism, Neuropsychopharmacology 30(1) (2005) 80-9.

[30] J.B. Chapman, M.G. Cutler, Sodium valproate: effects on social behaviour and physical development in the mouse, Psychopharmacology (Berl) 83(4) (1984) 390-6.

[31] S. Roux, Y. Bailly, J.L. Bossu, Regional and sex-dependent alterations in Purkinje cell density in the valproate mouse model of autism, Neuroreport 30(2) (2019) 82-88.

[32] B.S. Abrahams, D.H. Geschwind, Advances in autism genetics: on the threshold of a new neurobiology, Nature reviews. Genetics 9(5) (2008) 341-55.

[33] A.M. Persico, T. Bourgeron, Searching for ways out of the autism maze: genetic, epigenetic and environmental clues, Trends Neurosci 29(7) (2006) 349-58.

[34] J.D. Buxbaum, C. Betancur, O. Bozdagi, N.P. Dorr, G.A. Elder, P.R. Hof, Optimizing the phenotyping of rodent ASD models: enrichment analysis of mouse and human neurobiological phenotypes associated with high-risk autism genes identifies morphological, electrophysiological, neurological, and behavioral features, Molecular autism 3(1) (2012) 1.

[35] D.H. Geschwind, Autism: many genes, common pathways?, Cell 135(3) (2008) 391-5.

[36] E. Ey, C.S. Leblond, T. Bourgeron, Behavioral profiles of mouse models for autism spectrum disorders, Autism Res (2011).

[37] The Dutch-Belgian Fragile $X$ Consortium, Fmr1 knockout mice: a model to study fragile $X$ mental retardation, Cell 78(1) (1994) 23-33.

[38] M. Pieretti, F.P. Zhang, Y.H. Fu, S.T. Warren, B.A. Oostra, C.T. Caskey, D.L. Nelson, Absence of expression of the FMR-1 gene in fragile X syndrome, Cell 66(4) (1991) 817-22.

[39] S. Pietropaolo, E. Subashi, Mouse models of Fragile X syndrome, in: S. Pietropaolo, F. Sluyter, W.E. Crusio (Eds.), Behavioral Genetics of the Mouse, Cambridge University Press, Cambridge, 2014, pp. 146-63.

[40] D.B. Budimirovic, W.E. Kaufmann, What can we learn about autism from studying fragile $X$ syndrome?, Developmental neuroscience 33(5) (2011) 379-94. 
[41] D. Oddi, W.E. Crusio, F.R. D'Amato, S. Pietropaolo, Monogenic mouse models of social dysfunction: Implications for autism, Behav Brain Res (2013).

[42] C.A. Pardo, C.G. Eberhart, The neurobiology of autism, Brain Pathol 17(4) (2007) 434-47.

[43] C.E. McOmish, E.L. Burrows, A.J. Hannan, Identifying novel interventional strategies for psychiatric disorders: integrating genomics, 'enviromics' and gene-environment interactions in valid preclinical models, British journal of pharmacology 171(20) (2014) 4719-28.

[44] J. Nithianantharajah, A.J. Hannan, Enriched environments, experience-dependent plasticity and disorders of the nervous system, Nat Rev Neurosci 7(9) (2006) 697-709.

[45] H.Y. Zoghbi, M.F. Bear, Synaptic dysfunction in neurodevelopmental disorders associated with autism and intellectual disabilities, Cold Spring Harbor perspectives in biology 4(3) (2012).

[46] R. Delorme, E. Ey, R. Toro, M. Leboyer, C. Gillberg, T. Bourgeron, Progress toward treatments for synaptic defects in autism., Nat Med 19(6) (2013) 685-94.

[47] K.T.E. Kleijer, M. Schmeisser, D.D. Krueger, T.M. Boeckers, P. Scheiffele, T. Bourgeron, N. Brose, J.P.H. Burbach, Neurobiology of autism gene products: towards pathogenesis

and drug targets, Psychopharmacology 231 (2014) 1037-1062.

[48] K. Mackie, Distribution of cannabinoid receptors in the central and peripheral nervous system, Handbook of experimental pharmacology (168) (2005) 299-325.

[49] P. Berghuis, A.M. Rajnicek, Y.M. Morozov, R.A. Ross, J. Mulder, G.M. Urban, K. Monory, G. Marsicano, M. Matteoli, A. Canty, A.J. Irving, I. Katona, Y. Yanagawa, P. Rakic, B. Lutz, K. Mackie, T. Harkany, Hardwiring the brain: endocannabinoids shape neuronal connectivity, Science 316(5828) (2007) $1212-6$.

[50] T.F. Freund, I. Katona, D. Piomelli, Role of endogenous cannabinoids in synaptic signaling, Physiological reviews 83(3) (2003) 1017-66.

[51] K. Monory, B. Lutz, Genetic models of the endocannabinoid system, Current topics in behavioral neurosciences 1 (2009) 111-39. 
[52] L. Bellocchio, E. Soria-Gomez, C. Quarta, M. Metna-Laurent, P. Cardinal, E. Binder, A. Cannich, A. Delamarre, M. Haring, M. Martin-Fontecha, D. Vega, T. Leste-Lasserre, D. Bartsch, K. Monory, B. Lutz, F. Chaouloff, U. Pagotto, M. Guzman, D. Cota, G. Marsicano, Activation of the sympathetic nervous system mediates hypophagic and anxiety-like effects of $\mathrm{CB}(1)$ receptor blockade, Proc Natl Acad Sci U S A $110(12)(2013) 4786-91$.

[53] K. Monory, F. Massa, M. Egertova, M. Eder, H. Blaudzun, R. Westenbroek, W. Kelsch, W. Jacob, R. Marsch, M. Ekker, J. Long, J.L. Rubenstein, S. Goebbels, K.A. Nave, M. During, M. Klugmann, B. Wolfel, H.U. Dodt, W. Zieglgansberger, C.T. Wotjak, K. Mackie, M.R. Elphick, G. Marsicano, B. Lutz, The endocannabinoid system controls key epileptogenic circuits in the hippocampus, Neuron 51(4) (2006) 455-66.

[54] A. Busquets-Garcia, J. Bains, G. Marsicano, CB1 Receptor Signaling in the Brain: Extracting Specificity from Ubiquity, Neuropsychopharmacology 43(1) (2018) 4-20.

[55] L. Cristino, T. Bisogno, V. Di Marzo, Cannabinoids and the expanded endocannabinoid system in neurological disorders, Nat Rev Neurol 16(1) (2020) 9-29.

[56] V. Di Marzo, F. Piscitelli, The Endocannabinoid System and its Modulation by Phytocannabinoids, Neurotherapeutics 12(4) (2015) 692-8.

[57] C.J. Jordan, Z.X. Xi, Progress in brain cannabinoid CB2 receptor research: From genes to behavior, Neurosci Biobehav Rev 98 (2019) 208-220.

[58] C. Turcotte, M.R. Blanchet, M. Laviolette, N. Flamand, The CB2 receptor and its role as a regulator of inflammation, Cell Mol Life Sci 73(23) (2016) 4449-4470.

[59] M. Pistis, M. Melis, From surface to nuclear receptors: the endocannabinoid family extends its assets, Curr Med Chem 17(14) (2010) 1450-67.

[60] T. Rubino, E. Zamberletti, D. Parolaro, Endocannabinoids and Mental Disorders, Handbook of experimental pharmacology 231 (2015) 261-83.

[61] F.A. Iannotti, V. Di Marzo, S. Petrosino, Endocannabinoids and endocannabinoid-related mediators: Targets, metabolism and role in neurological disorders, Prog Lipid Res 62 (2016) 107-28. 
[62] E.M. Marco, G. Laviola, The endocannabinoid system in the regulation of emotions throughout lifespan: a discussion on therapeutic perspectives, J Psychopharmacol 26(1) (2012) 150-63.

[63] V. Trezza, R. Damsteegt, A. Manduca, S. Petrosino, L.W. Van Kerkhof, R.J. Pasterkamp, Y. Zhou, P. Campolongo, V. Cuomo, V. Di Marzo, L.J. Vanderschuren, Endocannabinoids in amygdala and nucleus accumbens mediate social play reward in adolescent rats, J Neurosci 32(43) (2012) 14899-908.

[64] L.K. Vaughn, G. Denning, K.L. Stuhr, H. de Wit, M.N. Hill, C.J. Hillard, Endocannabinoid signalling: has it got rhythm?, Br J Pharmacol 160(3) (2010) 530-43.

[65] B. Chakrabarti, L. Kent, J. Suckling, E. Bullmore, S. Baron-Cohen, Variations in the human cannabinoid receptor (CNR1) gene modulate striatal responses to happy faces, Eur J Neurosci 23(7) (2006) 1944-8.

[66] A.E. Purcell, O.H. Jeon, A.W. Zimmerman, M.E. Blue, J. Pevsner, Postmortem brain abnormalities of the glutamate neurotransmitter system in autism, Neurology 57(9) (2001) 1618-28.

[67] D.S. Karhson, K.M. Krasinska, J.A. Dallaire, R.A. Libove, J.M. Phillips, A.S. Chien, J.P. Garner, A.Y. Hardan, K.J. Parker, Plasma anandamide concentrations are lower in children with autism spectrum disorder, Molecular autism 9 (2018) 18.

[68] A. Aran, M. Eylon, M. Harel, L. Polianski, A. Nemirovski, S. Tepper, A. Schnapp, H. Cassuto, N. Wattad, J. Tam, Lower circulating endocannabinoid levels in children with autism spectrum disorder, Molecular autism 10 (2019) 2.

[69] D.R. Smith, C.M. Stanley, T. Foss, R.G. Boles, K. McKernan, Rare genetic variants in the endocannabinoid system genes CNR1 and DAGLA are associated with neurological phenotypes in humans, PLoS One 12(11) (2017) e0187926.

[70] D. Siniscalco, A. Sapone, C. Giordano, A. Cirillo, L. de Magistris, F. Rossi, A. Fasano, J.J. Bradstreet, S. Maione, N. Antonucci, Cannabinoid receptor type 2, but not type 1, is up-regulated in peripheral blood mononuclear cells of children affected by autistic disorders, J Autism Dev Disord 43(11) (2013) 2686-95. 
[71] S. Basu, B.N. Dittel, Unraveling the complexities of cannabinoid receptor 2 (CB2) immune regulation in health and disease, Immunol Res 51(1) (2011) 26-38.

[72] H. Ormstad, V. Bryn, O.D. Saugstad, O. Skjeldal, M. Maes, Role of the Immune System in Autism Spectrum Disorders (ASD), CNS Neurol Disord Drug Targets 17(7) (2018) 489-495.

[73] D. Siniscalco, S. Schultz, A.L. Brigida, N. Antonucci, Inflammation and Neuro-Immune Dysregulations in Autism Spectrum Disorders, Pharmaceuticals (Basel) 11(2) (2018).

[74] E. Zamberletti, M. Gabaglio, D. Parolaro, The Endocannabinoid System and Autism Spectrum Disorders: Insights from Animal Models, Int J Mol Sci 18(9) (2017).

[75] L. Zhang, B.E. Alger, Enhanced endocannabinoid signaling elevates neuronal excitability in fragile X syndrome, J Neurosci 30(16) (2010) 5724-9.

[76] M. Maccarrone, S. Rossi, M. Bari, V. De Chiara, C. Rapino, A. Musella, G. Bernardi, C. Bagni, D. Centonze, Abnormal mGlu 5 receptor/endocannabinoid coupling in mice lacking FMRP and BC1 RNA, Neuropsychopharmacology 35(7) (2010) 1500-9.

[77] K.M. Jung, M. Sepers, C.M. Henstridge, O. Lassalle, D. Neuhofer, H. Martin, M. Ginger, A. Frick, N.V. DiPatrizio, K. Mackie, I. Katona, D. Piomelli, O.J. Manzoni, Uncoupling of the endocannabinoid signalling complex in a mouse model of fragile X syndrome, Nature communications 3 (2012) 1080.

[78] R. Gomez, M. Navarro, B. Ferrer, J.M. Trigo, A. Bilbao, I. Del Arco, A. Cippitelli, F. Nava, D. Piomelli, F. Rodriguez de Fonseca, A peripheral mechanism for CB1 cannabinoid receptor-dependent modulation of feeding, J Neurosci 22(21) (2002) 9612-7.

[79] P. S., L.M. V., B. L., S. E., B. B., C. W.E., M. G., The endocannabinoid system critically modulates the expression of social behaviors in adult male mice, Society for Neuroscience (SFN) Annual Meeting New Orleans, USA, 2012.

[80] A. Busquets-Garcia, R. Maldonado, A. Ozaita, New insights into the molecular pathophysiology of fragile X syndrome and therapeutic perspectives from the animal model, The international journal of biochemistry \& cell biology 53 (2014) 121-6. 
[81] M. Gomis-Gonzalez, A. Busquets-Garcia, C. Matute, R. Maldonado, S. Mato, A. Ozaita, Possible Therapeutic Doses of Cannabinoid Type 1 Receptor Antagonist Reverses Key Alterations in Fragile X Syndrome Mouse Model, Genes (Basel) 7(9) (2016).

[82] M. Qin, Z. Zeidler, K. Moulton, L. Krych, Z. Xia, C.B. Smith, Endocannabinoid-mediated improvement on a test of aversive memory in a mouse model of fragile $X$ syndrome, Behav Brain Res 291 (2015) 164-171.

[83] D. Wei, D. Dinh, D. Lee, D. Li, A. Anguren, G. Moreno-Sanz, C.M. Gall, D. Piomelli, Enhancement of Anandamide-Mediated Endocannabinoid Signaling Corrects Autism-Related Social Impairment, Cannabis Cannabinoid Res 1(1) (2016) 81-89.

[84] G.G. Gould, A. Seillier, G. Weiss, A. Giuffrida, T.F. Burke, J.G. Hensler, C. Rock, A. Tristan, L.R. McMahon, A. Salazar, J.C. O'Connor, N. Satsangi, R.K. Satsangi, T.T. Gu, K. Treat, C. Smolik, S.T. Schultz, Acetaminophen differentially enhances social behavior and cortical cannabinoid levels in inbred mice, Prog Neuropsychopharmacol Biol Psychiatry 38(2) (2012) 260-9.

[85] G.G. Gould, T.F. Burke, M.D. Osorio, C.M. Smolik, W.Q. Zhang, E.S. Onaivi, T.T. Gu, M.N. DeSilva, J.G. Hensler, Enhanced novelty-induced corticosterone spike and upregulated serotonin 5-HT1A and cannabinoid CB1 receptors in adolescent BTBR mice, Psychoneuroendocrinology 39 (2014) 158-69.

[86] D. Wei, D. Lee, C.D. Cox, C.A. Karsten, O. Penagarikano, D.H. Geschwind, C.M. Gall, D. Piomelli, Endocannabinoid signaling mediates oxytocin-driven social reward, Proc Natl Acad Sci U S A 112(45) (2015) 14084-9.

[87] E.S. Onaivi, R. Benno, T. Halpern, M. Mehanovic, N. Schanz, C. Sanders, X. Yan, H. Ishiguro, Q.R. Liu, A.L. Berzal, M.P. Viveros, S.F. Ali, Consequences of cannabinoid and monoaminergic system disruption in a mouse model of autism spectrum disorders, Current neuropharmacology 9(1) (2011) 209-14.

[88] C. Foldy, R.C. Malenka, T.C. Sudhof, Autism-associated neuroligin-3 mutations commonly disrupt tonic endocannabinoid signaling, Neuron 78(3) (2013) 498-509. 
[89] H.E. Speed, I. Masiulis, J.R. Gibson, C.M. Powell, Increased Cortical Inhibition in Autism-Linked Neuroligin-3R451C Mice Is Due in Part to Loss of Endocannabinoid Signaling, PLoS One 10(10) (2015) e0140638.

[90] G. Martella, M. Meringolo, L. Trobiani, A. De Jaco, A. Pisani, P. Bonsi, The neurobiological bases of autism spectrum disorders: the R451C-neuroligin 3 mutation hampers the expression of long-term synaptic depression in the dorsal striatum, Eur J Neurosci 47(6) (2018) 701-708.

[91] S. Hosie, D.T. Malone, S. Liu, M. Glass, P.A. Adlard, A.J. Hannan, E.L. Hill-Yardin, Altered Amygdala Excitation and CB1 Receptor Modulation of Aggressive Behavior in the Neuroligin-3(R451C) Mouse Model of Autism, Frontiers in cellular neuroscience 12 (2018) 234.

[92] M. Servadio, F. Melancia, A. Manduca, A. di Masi, S. Schiavi, V. Cartocci, V. Pallottini, P. Campolongo, P. Ascenzi, V. Trezza, Targeting anandamide metabolism rescues core and associated autistic-like symptoms in rats prenatally exposed to valproic acid, Translational psychiatry 6(9) (2016) e902.

[93] D.M. Kerr, L. Downey, M. Conboy, D.P. Finn, M. Roche, Alterations in the endocannabinoid system in the rat valproic acid model of autism, Behav Brain Res 249 (2013) 124-32.

[94] D.M. Kerr, A. Gilmartin, M. Roche, Pharmacological inhibition of fatty acid amide hydrolase attenuates social behavioural deficits in male rats prenatally exposed to valproic acid, Pharmacol Res 113(Pt A) (2016) 228-235.

[95] S. Poleg, P. Golubchik, D. Offen, A. Weizman, Cannabidiol as a suggested candidate for treatment of autism spectrum disorder, Prog Neuropsychopharmacol Biol Psychiatry 89 (2019) 90-96.

[96] D. Barchel, O. Stolar, T. De-Haan, T. Ziv-Baran, N. Saban, D.O. Fuchs, G. Koren, M. Berkovitch, Oral Cannabidiol Use in Children With Autism Spectrum Disorder to Treat Related Symptoms and Comorbidities, Front Pharmacol 9 (2018) 1521.

[97] H. Heussler, J. Cohen, N. Silove, N. Tich, M.O. Bonn-Miller, W. Du, C. O'Neill, T. Sebree, A phase $1 / 2$, open-label assessment of the safety, tolerability, and efficacy of transdermal cannabidiol 
(ZYN002) for the treatment of pediatric fragile X syndrome, Journal of neurodevelopmental disorders 11(1) (2019) 16.

[98] N. Tartaglia, M. Bonn-Miller, R. Hagerman, Treatment of Fragile X Syndrome with Cannabidiol: A Case Series Study and Brief Review of the Literature, Cannabis Cannabinoid Res 4(1) (2019) 3-9.

[99] S. Burstein, Cannabidiol (CBD) and its analogs: a review of their effects on inflammation, Bioorg Med Chem 23(7) (2015) 1377-85.

[100] L. De Petrocellis, A. Ligresti, A.S. Moriello, M. Allara, T. Bisogno, S. Petrosino, C.G. Stott, V. Di Marzo, Effects of cannabinoids and cannabinoid-enriched Cannabis extracts on TRP channels and endocannabinoid metabolic enzymes, Br J Pharmacol 163(7) (2011) 1479-94.

[101] D. Vigli, L. Cosentino, C. Raggi, G. Laviola, M. Woolley-Roberts, B. De Filippis, Chronic treatment with the phytocannabinoid Cannabidivarin (CBDV) rescues behavioural alterations and brain atrophy in a mouse model of Rett syndrome, Neuropharmacology 140 (2018) 121-129.

[102] E. Zamberletti, M. Gabaglio, F. Piscitelli, J.S. Brodie, M. Woolley-Roberts, I. Barbiero, M. Tramarin, G. Binelli, N. Landsberger, C. Kilstrup-Nielsen, T. Rubino, V. Di Marzo, D. Parolaro, Cannabidivarin completely rescues cognitive deficits and delays neurological and motor defects in male Mecp2 mutant mice, J Psychopharmacol 33(7) (2019) 894-907.

[103] E. Zamberletti, M. Gabaglio, M. Woolley-Roberts, S. Bingham, T. Rubino, D. Parolaro, Cannabidivarin Treatment Ameliorates Autism-Like Behaviors and Restores Hippocampal Endocannabinoid System and Glia Alterations Induced by Prenatal Valproic Acid Exposure in Rats, Frontiers in cellular neuroscience 13 (2019) 367.

[104] A. Zehra, J. Burns, C.K. Liu, P. Manza, C.E. Wiers, N.D. Volkow, G.J. Wang, Cannabis Addiction and the Brain: a Review, J Neuroimmune Pharmacol 13(4) (2018) 438-452.

[105] K.L. Phan, M. Angstadt, J. Golden, I. Onyewuenyi, A. Popovska, H. de Wit, Cannabinoid modulation of amygdala reactivity to social signals of threat in humans, J Neurosci 28(10) (2008) 2313-9. 
[106] S. Pietropaolo, L. Bellocchio, A. Ruiz-Calvo, M. Cabanas, Z. Du, M. Guzman, M. Garret, Y.H. Cho, Chronic cannabinoid receptor stimulation selectively prevents motor impairments in a mouse model of Huntington's disease, Neuropharmacology 89 (2015) 368-74.

[107] M.G. Cutler, J.H. Mackintosh, Cannabis and delta-9-tetrahydrocannabinol. Effects on elements of social behaviour in mice, Neuropharmacology 23(9) (1984) 1091-7.

[108] M. Dorr, H. Steinberg, Effects of delta9-tetrahydrocannabinol on social behaviour in mice: comparison between two vehicles, Psychopharmacology (Berl) 47(1) (1976) 87-91.

[109] D.L. Ely, J.P. Henry, C.J. Jarosz, Effects of marihuana (delta9-THC) on behavior patterns and social roles in colonies of CBA mice, Behavioral biology 13(3) (1975) 263-76.

[110] L.E. Long, R. Chesworth, J.C. Arnold, T. Karl, A follow-up study: acute behavioural effects of Delta(9)-THC in female heterozygous neuregulin 1 transmembrane domain mutant mice, Psychopharmacology (Berl) 211(3) (2010) 277-89.

[111] B. Sieber, H.R. Frischknecht, P.G. Waser, Behavioral effects of hashish in mice. IV. Social dominance, food dominance, and sexual behavior within a group of males, Psychopharmacology (Berl) 73(2) (1981) 142-6.

[112] L. Bellocchio, P. Lafenetre, A. Cannich, D. Cota, N. Puente, P. Grandes, F. Chaouloff, P.V. Piazza, G. Marsicano, Bimodal control of stimulated food intake by the endocannabinoid system, Nat Neurosci 13(3) (2010) 281-3.

[113] S. Ruehle, A.A. Rey, F. Remmers, B. Lutz, The endocannabinoid system in anxiety, fear memory and habituation, J Psychopharmacol 26(1) (2012) 23-39.

[114] M. Haring, V. Enk, A. Aparisi Rey, S. Loch, I. Ruiz de Azua, T. Weber, D. Bartsch, K. Monory, B. Lutz, Cannabinoid type-1 receptor signaling in central serotonergic neurons regulates anxiety-like behavior and sociability, Front Behav Neurosci 9 (2015) 235.

[115] M. Haring, N. Kaiser, K. Monory, B. Lutz, Circuit specific functions of cannabinoid CB1 receptor in the balance of investigatory drive and exploration, PLoS One 6(11) (2011) e26617. 
[116] A.L. Terzian, V. Micale, C.T. Wotjak, Cannabinoid receptor type 1 receptors on GABAergic vs. glutamatergic neurons differentially gate sex-dependent social interest in mice, Eur J Neurosci 40(1) (2014) 2293-8.

[117] S. Rossi, V. De Chiara, A. Musella, L. Sacchetti, C. Cantarella, M. Castelli, F. Cavasinni, C. Motta, V. Studer, G. Bernardi, B.F. Cravatt, M. Maccarrone, A. Usiello, D. Centonze, Preservation of striatal cannabinoid CB1 receptor function correlates with the antianxiety effects of fatty acid amide hydrolase inhibition, Molecular pharmacology 78(2) (2010) 260-8.

[118] N. Gonczarowska, C. Tomaz, F.V. Caixeta, R. Malcher-Lopes, M. Barros, H. Nishijo, R.S. Maior, CB1 receptor antagonism in capuchin monkeys alters social interaction and aversive memory extinction, Psychopharmacology (Berl) 236(12) (2019) 3413-3419.

[119] A. Seillier, A.A. Martinez, A. Giuffrida, Phencyclidine-induced social withdrawal results from deficient stimulation of cannabinoid $\mathrm{CB}(1)$ receptors: implications for schizophrenia, Neuropsychopharmacology 38(9) (2013) 1816-24.

[120] Y. Litvin, A. Phan, M.N. Hill, D.W. Pfaff, B.S. McEwen, CB1 receptor signaling regulates social anxiety and memory, Genes, brain, and behavior 12(5) (2013) 479-89.

[121] J. Gaudissard, M. Ginger, M. Premoli, M. Memo, A. Frick, S. Pietropaolo, Behavioral abnormalities in the Fmr1-KO2 mouse model of fragile $\mathrm{X}$ syndrome: The relevance of early life phases, Autism Res 10(10) (2017) 1584-1596.

[122] D. Oddi, E. Subashi, S. Middei, L. Bellocchio, V. Lemaire-Mayo, M. Guzman, W.E. Crusio, F.R. D'Amato, S. Pietropaolo, Early social enrichment rescues adult behavioral and brain abnormalities in a mouse model of fragile X syndrome, Neuropsychopharmacology 40(5) (2015) 1113-22.

[123] J. Haller, B. Varga, C. Ledent, T.F. Freund, CB1 cannabinoid receptors mediate anxiolytic effects: convergent genetic and pharmacological evidence with CB1-specific agents, Behav Pharmacol 15(4) (2004) 299-304. 
[124] A.L. Terzian, F. Drago, C.T. Wotjak, V. Micale, The Dopamine and Cannabinoid Interaction in the Modulation of Emotions and Cognition: Assessing the Role of Cannabinoid CB1 Receptor in Neurons Expressing Dopamine D1 Receptors, Front Behav Neurosci 5 (2011) 49.

[125] W. Fyke, M. Premoli, V. Lemaire-Mayo, S. Middei, G. Marsicano, W.E. Crusio, S.U.c.i.t.C.k.m.I.d.d.a.a.a. Pietropaolo, Ultrasonic communication in the CB1 knockout mouse line during development and at adulthood. , European Brain and Behaviour Society Annual Meeting, Prague, Czech Republic, 2019.

[126] M.L. Yeh, E.S. Levine, Perspectives on the Role of Endocannabinoids in Autism Spectrum Disorders, OBM Neurobiol 1(2) (2017).

[127] Z. Wen, H.N. Nguyen, Z. Guo, M.A. Lalli, X. Wang, Y. Su, N.S. Kim, K.J. Yoon, J. Shin, C. Zhang, G. Makri, D. Nauen, H. Yu, E. Guzman, C.H. Chiang, N. Yoritomo, K. Kaibuchi, J. Zou, K.M. Christian, L. Cheng, C.A. Ross, R.L. Margolis, G. Chen, K.S. Kosik, H. Song, G.L. Ming, Synaptic dysregulation in a human iPS cell model of mental disorders, Nature 515(7527) (2014) 414-8.

[128] F. Happe, Cognition in autism: one deficit or many?, Novartis Foundation symposium 251 (2003) 198-207; discussion 207-12, 281-97.

[129] S. Pietropaolo, W.E. Crusio, R. D'Amato F, Treatment Approaches in Rodent Models for Autism Spectrum Disorder, Current topics in behavioral neurosciences 30 (2017) 325-340.

[130] C. Gross, E.M. Berry-Kravis, G.J. Bassell, Therapeutic strategies in fragile X syndrome: dysregulated mGluR signaling and beyond, Neuropsychopharmacology : official publication of the American College of Neuropsychopharmacology 37(1) (2012) 178-95. 